\title{
Diving deep into chelation therapy for coronary artery disease: a review
}

\author{
Iwan Cahyo Santosa Putra, William Kamarullah*
}

Faculty of Medicine and Health Science, Atma Jaya Catholic University of Indonesia, Indonesia

Received: 14 October 2019 Accepted: 16 November 2019

*Correspondence to: Dr. William Kamarullah, Email: williamkamarullah@ hotmail.com

Copyright: (C) the author(s), publisher and licensee Medip Academy. This is an openaccess article distributed under the terms of the Creative Commons Attribution NonCommercial License, which permits unrestricted noncommercial use, distribution, and reproduction in any medium, provided the original work is properly cited.

\begin{abstract}
Chelation therapy is still a mainstay therapy when it comes to dealing with heavy metal intoxication. The ability of various chelators to bind metal and other chemical molecules led to the idea whether chelation therapy can be used as an alternative therapy to enchain calcium element that is known to be present in the atherosclerotic plaque. Various studies have been conducted, one of which is a large trial to assess chelation therapy study to show in case ethylenediaminetetraacetic acid (EDTA) chelator can be proven by evidencebased in managing coronary heart disease. Despite the favorable results that were found in many literature studies, the results of the study are still under debate in various aspects. To address this issue, we conducted a review article to discuss comprehensively the general description of EDTA as chelation therapy, various mechanisms that can explain the use of EDTA in the management of coronary heart disease, the pharmacokinetic aspects of EDTA chelation therapy, as well as describing various existing studies with a good level of evidence to review the effectiveness of these therapies against coronary artery disease.
\end{abstract}

Keywords: Chelation therapy, EDTA, Cardiovascular disease, Coronary artery disease

\section{INTRODUCTION}

Coronary artery disease is a common term for the buildup of plaque in the heart's arteries that could lead to heart attack. ${ }^{1}$ While this condition is still the leading cause of death in the world, in fact, it accounts for $35 \%$ of causes of death in Indonesia. ${ }^{2}$ As a result of highly resulting mortality, many alternative therapeutic options offered by various clinicians in the world in order to reduce the incidence of recurrence and global mortality rates of this condition. One alternative therapy that is said to be effective in treating and controlling coronary heart disease by various practitioners in the world of medicine is chelation therapy. Chelation therapy is a therapy that is generally used as a treatment for metal poisoning. This therapy involves a process of administering a substance, so-called chelator which functions as a metal binding agent in the bloodstream so that the metal can be excreted in urine. One chelator that has been approved by the Food and Drug Administration (FDA) in managing lead poisoning is ethylenediaminetetraacetic acid (EDTA). Alternative medicine practitioners have been using a similar chelator, disodium EDTA, to treat heart disease, claiming to see benefits. Nevertheless, according to the CDC, there were reportedly 111 thousand adults and 72 thousand children under 18 years using chelation therapy with uncertain indications, which create controversies. ${ }^{3,4}$ As a result of various controversies regarding the efficacy 
of chelation therapy in treating coronary heart disease, the National Heart, Lung, and Blood Institute and the National Center for Complementary and Alternative Medicine issued a request for further research to be conducted about chelation therapy. Therefore, a doubleblind, placebo-controlled study called the trial to assess chelation therapy (TACT), was developed and funded in 2002 to evaluate disodium EDTA chelation therapy for patients who had a previous myocardial infarction, despite the resulting decisions are not favorable enough to provide the evidence. ${ }^{5}$ In this review article, a general overview of chelation therapy will be discussed, as well as the mechanism of action of chelation therapy, pharmacokinetic aspects, and several recent human studies on the efficacy of chelation therapy in managing coronary artery disease.

\section{CHELATING AGENTS: AN OVERVIEW}

\section{Brief chronological history}

Chelation comes from the Greek word "Chele", which means it resembles a crab or lobster claw. The history of the use of chelation therapy began to be known in the late $19^{\text {th }}$ century and early $20^{\text {th }}$ century, where several chemical agents were believed to be able to effectively extract and bind metals and minerals. ${ }^{6}$ Understanding the ability of various chemical agents which capable of binding and removing heavy metals in the human body system adds new insights into medical science, especially in the field of therapeutic applications. It should be understood that the term chelation is generally a reaction term between the ion of a charged element and an agent, so-called chelator, whether it is from a plant-derived source or an administered pharmaceutical agent. ${ }^{7}$

Ethylenediaminetetraacetic acid (disodium EDTA), first introduced by Ferdinand Munz in 1938, is one of the chelators capable of binding various types of metals, such as lead, cadmium, and even calcium, so that in the early $20^{\text {th }}$ century, motivated by the calcification present in advanced coronary disease, practitioners began using EDTA to treat symptoms of cardiovascular disease with initially positive, uncontrolled albeit, results. ${ }^{8}$ A chemist named Frederick Bersworth found that EDTA works on calcium homeostasis systems, thus making the chelator can be used as an anticoagulant in laboratory settings. Thus, this postulate is garnering attention to place chelation therapy as one of the alternative therapies for a cardiovascular disease. ${ }^{7}$

\section{EDTA: The main chelator for coronary artery disease}

A chemical molecule, both organic and non-organic, has the ability to form two or more stable bonds with metal atoms, or what can be called as chelating agents. Etymologically, chelation comes from the Greek word, chele, which means claw. This shows that this claw-like molecular structure can increase the affinity of chemical bonds that are stronger than molecules that are non- chelating. As a result of this, many chelators are made synthetically for certain medical treatment purposes. ${ }^{9}$<smiles>O=C(O)CN(CCN(CC(=O)O)CC(=O)O)CC(=O)O</smiles>

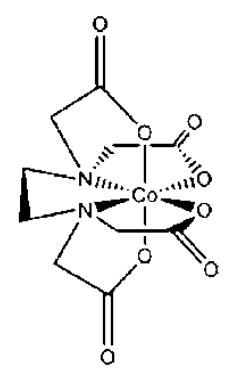

Figure 1: The chemical structure of EDTA; it is a polyprotic acid containing four carboxylic acid and two amine groups with lone pair electrons. ${ }^{10}$

One of the synthesized chelators is EDTA. EDTA is an amino acid that is synthesized with strong chelation bonds with valences range from +2 to +6 . The EDTAmetal chelate is a water-soluble substance and typically excreted in the urine. It can form strong covalent bonds with some xenobiotic metals and increase the urinary excretion of calcium, zinc, cadmium, and lead. Its affinity to calcium led to the early hypothesis that EDTA might decalcify the atherosclerotic plaque so that it can be used as an alternative coronary artery disease treatment. ${ }^{11}$

\section{ROLE OF CHELATION THERAPY AS A CORONARY ARTERY DISEASE TREATMENT}

As stated before, EDTA is a synthetic amino acid that has a high affinity for binding to divalent or trivalent cations, such as magnesium, lead, zinc, iron, and calcium. In general, EDTA will bind to several cations that can be bound and convert it into a redox inactive state and excreted from the body through urine. As a result of the frequent discovery of calcium in atherosclerotic plaques, many hypotheses say that EDTA might be able to effectively overcome atherosclerosis. ${ }^{12}$

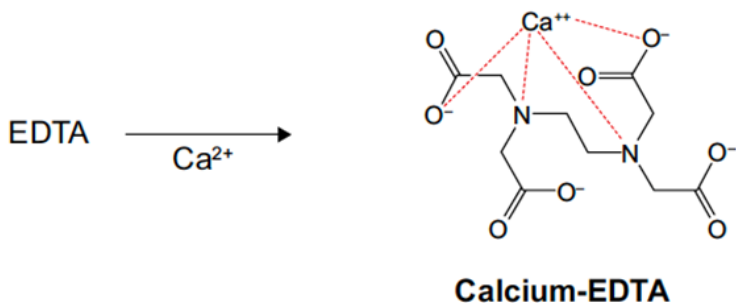

Figure 2: The molecular structure of calcium chelated with EDTA; EDTA is a versatile agent that can form four or six bonds with a metal ion; in the calcium complex, $[\mathrm{Ca}(\text { EDTA })]^{2-}$, EDTA is a tetradentate ligand, and chelation involves two nitrogen atoms and two oxygen atoms. ${ }^{13}$

EDTA is thought to have several mechanisms in dealing with coronary heart disease, including binding to calcium 
as an element in atheromatous plaques (as stated in Figure 2), as an antioxidant to prevent lipid oxidation, reduce iron reserves in the body, and arterial dilation. ${ }^{14}$

\section{Antioxidant properties}

Chelation therapy is thought to not only remove contaminating metals but also to decrease free radical production. ${ }^{15}$ The pathogenesis of atherosclerosis begins with the process of peroxidation of PUFA (polyunsaturated fatty acids) which will turn into oxidatively modified lipoprotein. The resulting lipid peroxide radical can then propagate the radical reaction by abstracting hydrogens from neighboring phospholipids or can react with itself to create a large number of secondary peroxidation products which are relevant to atherogenesis. ${ }^{16}$

An experimental study was conducted by Roussel et al to prove whether multiple sessions of EDTA chelation therapy decrease oxidative stress. As one of the biomarkers for lipid peroxidation process, plasma malondialdehyde (MDA) concentrations were assessed using high-performance liquid chromatography (HPLC) in the study. It's reported that chelation therapy with EDTA shows a protective effect on the lipid peroxidation process. This was indicated by a decrease in plasma MDA levels after ten sessions of EDTA chelation therapy interventions for five weeks $(2.54 \pm 0.44 \mathrm{mmol} / \mathrm{l}$ (before) to $2.02 \pm 0.26 \mathrm{mmol} / \mathrm{l}$ (after 10 sessions $))(\mathrm{p}<0.05) .{ }^{15}$

In addition, a comorbid factor such as diabetes mellitus can cause the accumulation of advanced glycation end products. AGEP requires metal catalyzed oxygen chemistry for their formation which binds to glycation end-products and promote the formation of reactive oxygen species in an autocatalytic reaction. This formation accumulates in tissues and promotes inflammation and oxidative stress as hallmarks of atherosclerosis. The benefits of the multicomponent EDTA-based infusion may be mediated through the chelation of metals, thus, reducing the inflammation and oxidative stress. $^{17}$

\section{Reducing iron reserves in the body}

Atherosclerosis is a chronic fibroproliferative inflammatory process and iron, through increased oxidation stress as well as directly, can control both native and adaptive immune responses. Within the arterial wall, iron affects all of the cell types that participate in the atherosclerotic process (monocytes/macrophages, endothelial cells, vascular smooth muscle cells and platelets). As the key cellular effectors of innate immunity, monocytes are attracted to activated endothelial cells by chemoattractant, such as macrophage chemoattractant protein-1 (MCP-1). Circulating monocytes adhere to the endothelial surface, enter the of the arterial intima, become activated by macrophage-colony stimulating factor (M-CSF), differentiate into macrophages, and then transform into foam cells, a type of cells that contain cholesterol. These can form a plaque that can lead to atherosclerosis. ${ }^{18}$

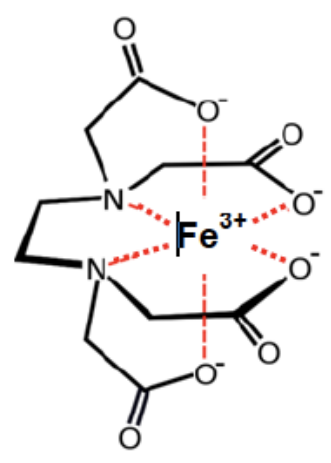

Figure 3: Chemical structure of ferric EDTA [Fe(EDTA) $]^{-}$; basically, iron in human blood plasma is more in the form of $\mathrm{Fe}^{3+}$; four oxygen atoms and two nitrogen atoms bond to the ferric ion in an octahedral arrangement; these complexes are often called chelates. ${ }^{19}$

With that knowledge, as EDTA has the ability to chelate iron, this is one of the reasons for the hypothesis that chelation therapy can be used to treat atherosclerosis as a predisposing factor for coronary artery disease. ${ }^{18}$

\section{Dilatation of artery}

There is also growing evidence that the depression of NO-related, endothelium-dependent vasodilation associated with vascular disease and with various vascular risk factors is largely due to an increase in the inactivation of NO by oxygen free radicals. ${ }^{20}$ EDTA is said to affect endothelial cells to produce NO and it is probably done through its oxidant mechanisms. It is also believed arterial dilation due to possible calcium channel blocking actions, or stimulation of prostacyclin production and improvement in arterial wall elasticity. ${ }^{12}$

\section{PHARMACOKINETIC PROFILE}

EDTA is a molecule that is difficult to absorb through the digestive tract $(<5 \%){ }^{21}$ It is also reported that the absorption of harmful substances such as lead will increase in the digestive tract along with the presence of EDTA in the digestive tract because the lead chelate formed will be more soluble from lead itself, so parenteral administration is a better option. ${ }^{21}$ It is primarily distributed in extracellular fluids $(90 \%)$ so that its ability to conduct chelation process in intracellular and cerebrospinal fluid (CSF) is very limited. EDTA also binds tightly to essential nutritional minerals (zinc, copper, manganese, chromium, and others) and trace elements from food and from nutritional supplements, blocking their absorption, and leading us to face serious nutritional deficiencies. ${ }^{21,22}$ 
There are two types of EDTA approved by the FDA, namely calcium disodium EDTA and disodium EDTA. Calcium disodium EDTA is approved by the FDA for use in lead poisoning and has been the mainstay of treatment for childhood lead poisoning. The second drug, disodium EDTA, is approved for use in patients with rhythm disorders from drug intoxication such as digitalis where there is hypercalcemia. ${ }^{8}$ After intravenous injection, the plasma half-life of EDTA is 20 to 60 minutes with the maximum safe dose $50 \mathrm{mg} / \mathrm{kg} /$ day and the maximum infusion rate is $16.6 \mathrm{mg} / \mathrm{min}$ over 3 hours. No metabolism occurs within both of the subtype and are excreted in the urine either unchanged or as the metal chelates (in the first hour after administration, half of the product is excreted in the urine, $70 \%$ or more during first 4 hours, and $95 \%$ in 24 hours). ${ }^{22-24}$

\section{CURRENTLY AVAILABLE HUMAN STUDIES}

The relationship between chelation therapy with coronary heart disease has been extensively investigated both in terms of the underlying mechanism and from the pharmacological aspects. Nevertheless, the efficacy of chelation therapy for cardiovascular disease is still being debated. Referring to the era of evidence-based medicine, in this review, we will present some of the results of currently available human studies regarding the effectiveness of chelation therapy in the management of coronary artery disease

\section{Methods}

We use Google Scholar and PubMed search engines to find studies that discuss the effect of chelation therapy on cardiovascular disease, specifically on coronary heart disease. Our literature search is arranged so that the literature that appears is in the range of 2000 to August 2019 with keywords, such as "chelation therapy", "EDTA", "cardiovascular disease", "coronary artery disease", "treatment".

\section{Literature review results}

The first literature study we found was a double-blind randomized controlled trial conducted in 2003 by Anderson et al. ${ }^{25}$ The purpose of this study was to evaluate the usefulness of chelation on endotheliumdependent vasomotor responses in patients with documented coronary artery disease (CAD). This study has 47 respondents who suffer from CAD. The diagnosis of CAD is established by a treadmill test in which electrocardiogram patients show ST depression in the 2 to 14 minutes from the beginning of the exercise. Respondents will be divided into placebo groups and therapeutic groups. Therapeutic group was given $500 \mathrm{ml}$ infusion solution of $5 \%$ dextrose in water for the active treatment containing disodium EDTA. The dose of EDTA was weight adjusted $(40 \mathrm{mg} / \mathrm{kg} \mathrm{BW})$, with the maximum total dose for each treatment is $3 \mathrm{~g}$. Each treatment solution also contained $750 \mathrm{mg}$ of magnesium sulfate, $5 \mathrm{~g}$ of ascorbic acid, and $5 \mathrm{~g}$ of sodium bicarbonate in the D5W. The infusion solution was administered over 3 hours to minimize the potential unblinding effect of infusion-related side effects. All patients received treatments twice weekly for 15 weeks and once per month for an additional three months (a total of 33 treatments). In the placebo group, $20 \mathrm{ml}$ of $0.9 \% \mathrm{NaCl}$ will be given. Both groups also received oral multivitamin therapy. At the end of the study, it was found that EDTA chelation therapy in combination with vitamins and minerals does not provide additional benefits on abnormal vasomotor responses in patients with $\mathrm{CAD}$ optimally treated with proven therapies for atherosclerotic risk factors. ${ }^{25}$

After so many studies were conducted to assess the effectiveness of EDTA-chelation therapy in managing CAD, the Cochrane Database of Systematic Reviews also published results for the effects of EDTA chelation therapy on clinical outcomes among people with atherosclerotic cardiovascular disease. A total of seven randomized controlled trials have been collected in this systematic review with participants with peripheral vascular disease (two of the seven studies were actually sub-studies of another). Outcomes measured in this study are digital subtraction angiogram, walking distance based on the onset of pain or disabling claudication, subjective walking distance, ankle brachial pressure index (ABPI), lifestyle measures (such as quality of life and physical well-being), blood lipids levels, pain-free maximal walking distance measured on a treadmill, or walking and bicycle stress tests. Mortality was not reported in any of the trials. Non-fatal events such as myocardial infarction, unstable angina pectoris and cerebrovascular events also were not included as endpoints in any of the studies. All studies compared EDTA to placebo. The placebo was either an isotonic solution or distilled water. Overall, the studies analyzed in this review did not show that chelation therapy has a statistically beneficial effect on patients who have peripheral vascular disease. A single study showed benefits, but conclusions were weak because of serious methodological flaws seen upon review (Olszewer et al). ${ }^{26,27}$

A double-blind, randomized controlled trial conducted by Knudtson et al also showed similar results. In this study, 84 patients aged $\geq 21$ years who had coronary artery disease (diagnosed with angiography or documented MI) were conducted. In this study, it was divided into two groups, namely the placebo group (43 participants) and the chelation therapy intervention group (41 participants). In the intervention group, participants were given $500 \mathrm{ml}$ infusion solution of $5 \%$ dextrose in water containing disodium EDTA and was weight adjusted (40 mg/ $\mathrm{kg}$ ), with a maximum total dose for each treatment of $3 \mathrm{~g}$. Each treatment solution also contained $750 \mathrm{mg}$ of magnesium sulfate, $5 \mathrm{~g}$ of ascorbic acid, and $5 \mathrm{~g}$ of sodium bicarbonate (titrated to physiologic $\mathrm{pH}$ ) in the $5 \%$ dextrose. In the placebo infusion solution, the EDTA was replaced by $20 \mathrm{ml}$ of $0.9 \%$ sodium chloride. As for the study end points, exercise parameters and quality of life 
questionnaires were collected at 15 and 27 weeks after randomization. The primary end point was the change in time to reach at least $1 \mathrm{~mm}$ of ST-segment depression at the 27-week evaluation. After the statistical analysis, it is reported that no beneficial effect of chelation therapy was found in patients with ischemic heart disease, stable angina, and positive treadmill test for ischemia. ${ }^{28}$

\section{Trial to assess chelation therapy (TACT)}

After some of the reviews that have been described above, TACT was the largest randomized clinical trial evaluating disodium EDTA in 1708 patient post-myocardial infarction. TACT was a double-blind $2 \times 2$ factorial trial: patients were randomized to receive 40 infusions of disodium EDTA chelation or placebo and additionally to an oral high-dose vitamin and mineral regimen or placebo. Details of the study protocol can be seen in Figure 4. ${ }^{29}$

Eligible patients were at least 50 years old and had experienced a myocardial infarction (MI) 6 weeks or more prior to enrollment. Among the 1708 participants who were randomly drawn, were divided into two further groups, namely the group receiving EDTA chelation (839 participants) and the group receiving placebo infusion (869 participants). Between the two groups, they were furtherly divided into 4 groups, namely chelation therapy with additional vitamins and minerals, chelation therapy with additional vitamins and minerals (placebo), placebo therapy with additional vitamins and minerals, and placebo therapy with additional vitamins and minerals (placebo). For the intervention group, patients were randomized to receive 40 infusions of a $500-\mathrm{ml}$ chelation solution (3 $\mathrm{g}$ of disodium EDTA, $7 \mathrm{~g}$ of ascorbate, $\mathrm{B}$ vitamins, electrolytes, procaine, and heparin). For the placebo group, the infusion consists of $500 \mathrm{ml}$ of normal saline and $1.2 \%$ dextrose $(2.5 \mathrm{~g}$ total). Each group also added an oral vitamin-mineral regimen vs an oral placebo. Infusions were administered weekly for 30 weeks, followed by 10 infusions 2 to 8 weeks apart. The prespecified primary end point was a composite of total mortality, recurrent MI, stroke, coronary revascularization, or hospitalization for angina. ${ }^{29}$

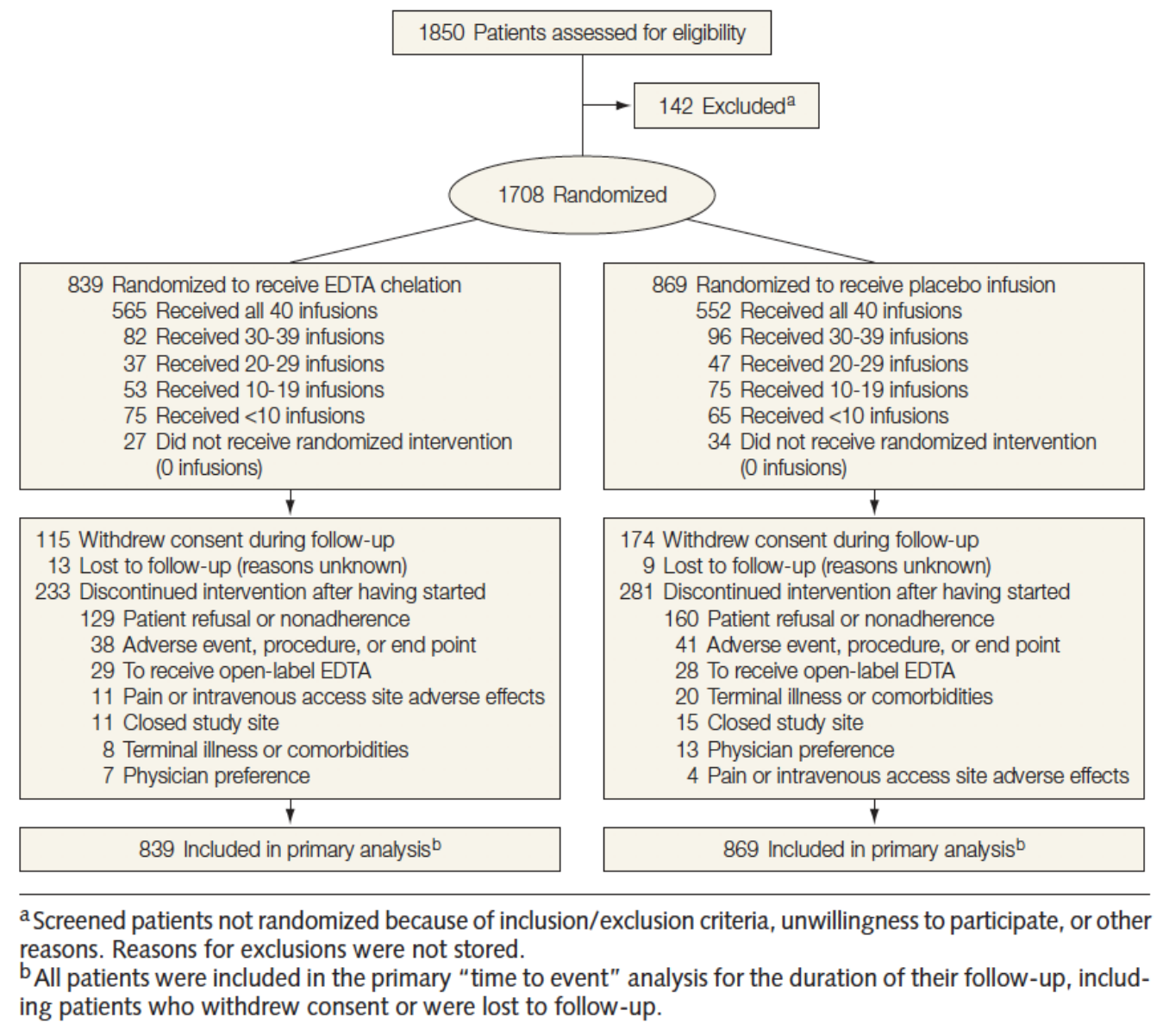

Figure 4: The study protocol and the participant flow in TACT study. ${ }^{29}$

TACT also pre-specified several subgroups for analyses based on assessing underrepresented populations (women and minorities), elderly persons (aged >70 years), high- risk patients (MI location, diabetes, and metabolic syndrome), and other subgroups of interest (time from index MI to trial enrollment, patients in whom statin therapy was not being used). ${ }^{29}$ 
In the primary end points analysis between the two study groups (EDTA chelation vs. placebo) there were 222 participants $(26 \%)$ in the EDTA group and 261 participants $(30 \%)$ in the placebo group who experienced mortality, recurrent MI, stroke, coronary revascularization, or hospitalization for angina with hazard ratio (HR) 0.82 (meaning that at any time, $82 \%$ of participants in the treatment group are having an event proportionally to the placebo group), 95\% CI: 0.69-0.99, $\mathrm{p}=0.035$. $^{28,30}$ If those two groups were divided again into the subgroups that have been mentioned above, there are two subgroups that have beneficial results, namely participants with anterior wall myocardial infarction and participants who suffer from diabetes. Participants who had anterior myocardial wall infarction had a reduced risk of primary end points with $\mathrm{HR}$ of $0.63,95 \% \mathrm{CI}$ : 0.47 $0.86, p=0.003$. In addition, participants who had diabetes also had a reduced risk of primary end points with HR of $0.61,95 \%$ CI: $0.45-0.83, \mathrm{p}=0.002{ }^{29}$

\section{Chelation therapy on post-MI patients with diabetes}

In relation to the TACT results that have been described above, it is stated that chelation therapy has beneficial results especially in the subgroup of participants who have diabetes. Thus, Ujueta et al conducted further analysis on the results of the TACT study, especially in participants with diabetes. The diabetes mellitus subgroup was defined as having self-reported diabetes mellitus, was taking oral or insulin treatment for diabetes mellitus, or had fasting blood glucose of at least $126 \mathrm{mg} / \mathrm{dl}$ at the time of enrollment in the study. The primary end points for this study are the same as TACT. As for the results, the incidence of the primary end-point was significantly lower in post-MI patients with diabetes treated with chelation therapy compared with placebo (HR 0.68, 95\% CI: 0.48-0.97, $\mathrm{p}=0.035){ }^{31}$ AHA (American Heart Association) also stated that patients with post-MI diabetes mellitus aged $\geq 50$ years on evidence-based medications demonstrated a marked reduction in cardiovascular events but do not constitute sufficient evidence to indicate the routine use of chelation therapy for all patients with post-MI diabetes mellitus (Recommendation class IIb (level of evidence B)). ${ }^{17}$

\section{Chelation therapy alone vs. in combination with oral high-dose multivitamins (with and without statins)}

In connection with the significant benefits of chelation therapy for the diabetes subpopulation found in the TACT study and regarding the number of variables that can be furtherly analyzed in the study, we tried to find a study within the diabetes population in TACT study which were analyzed using other variables. In the TACT study, it was written that the chelation practitioners argued forcefully during the design phase of TACT for the inclusion of an adjunctive high-dose vitamin and mineral regimen. Thus, Lamas et al furtherly analyzed the TACT study by adding 6 caplets daily of a 28-component multivitaminmultimineral mixture or placebo. The study protocol also added the well-known pre-specified subgroups such as diabetes population within it. A total of 421 participants were in the EDTA chelation infusions + high-dose oral multivitamins group, 418 participants in the EDTA chelation infusions + oral placebo group, 432 participants in the placebo infusions + high dose oral multivitamins group, and 437 participants in the placebo infusions + oral placebo group. The median duration of follow-up was 55 months overall. However, there was no significant difference in the length of follow-up across all 4 groups. Whereas in 631 participants in the diabetes population, a statistically significant result was found that the primary end point by treatment group occurred in 56 (38\%) of the placebo infusion + placebo vitamin group and 36 (23\%) of patients in the chelation + high-dose vitamin group (HR 0.49 , 95\% CI: 0.33-0.75, p<0.001). Nonetheless, the other two groups benefit not statistically significantly difference. $^{32}$

Furthermore, there's also a study that analyzed participants who received a high-dose complex oral multivitamins and multi-mineral regimen versus placebo but weren't on statin therapy at baseline in the TACT study. In this study, there were 460 participants not taking statins at the baseline of the study (224 of them were in the active vitamin group and 236 of them were in the placebo group). As for the non-statin participants, the primary end point of the study occurred in 137 participants, of which 51 participants were from the active vitamin group and 86 participants were from the placebo group (HR 0.62, 95\% CI: $0.44-0.87, \mathrm{p}=0.006){ }^{33}$

\section{Trial to assess chelation therapy 2 (TACT2)}

Although the various studies that have been produced from TACT have yielded many meaningful results, the shortcomings of this study must also be reviewed to reduce bias. First, regarding participants who rejected informed consent, $13.7 \%$ of participants and $20 \%$ of participants refused in the intervention group and the placebo group respectively. This is an indication of the term, unblinding. In addition, this study was also unable to explain the mechanism by which chelation therapy can reduce cardiovascular events. Moreover, this study did not ask any further about the history of heavy metal exposure, making it difficult for the medical community to interpret the results of the study. Furthermore, this study also included several sites specifically focusing on the field of alternative medicines (60\%) and had not previously carried out clinical trials, so the reliability of the study results was still in doubt. ${ }^{34}$

Having known the various limitations and shortcomings of the TACT study, making it warrants for further research. One of the implementations that we can observe is the enactment of TACT2 study. As a further form of TACT study results, TACT2 is a randomized, double blind controlled factorial clinical trial of edited disodiumbased chelation and high-dose oral vitamins and minerals to prevent recurrent cardiac events in diabetes patients 
with prior myocardial infarction (MI). So far, there are 1200 participants with diabetes and a history of myocardial infarction have been collected and are expected to be completed by September $2020 .^{35}$

\section{CONCLUSION}

From the results of literature studies that have been done, theoretically, it can be seen that chelation therapy has a series of mechanisms in treating cardiovascular disease, especially in coronary artery disease. Although various studies, especially TACT, show significant results regarding chelation therapy for coronary heart disease, this study still has many shortcomings and the reliability of the results is still doubtful. Thus, there is currently not enough evidence to recommend routine use of chelation therapy in coronary artery disease. Hopefully, some future studies can provide such satisfying results.

Funding: No funding sources

Conflict of interest: None declared

Ethical approval: Not required

\section{REFERENCES}

1. American Heart Association - Coronary Artery Disease. Available at: https://www.heart.org/ en/health-topics/consumer-healthcare/what-iscardiovascular-disease/coronary-artery-disease. Accessed on 01 September 2019.

2. World Health Organization - Noncommunicable Disease (NCD) Country Profiles (Indonesia). Available at: https://www.who.int/nmh/countries/ idn_en.pdf. Accessed on 01 September 2019.

3. American Heart Association - Chelation Therapy: A New Look at an Old Treatment for Heart Disease, Particularly in Diabetics. Available at: https://www. ahajournals.org/doi/10.1161/CIRCULATIONAHA.1 14.010774. Accessed on 01 September 2019.

4. Deaths Associated with Hypocalcemia from Chelation Therapy --- Texas, Pennsylvania, and Oregon, 2003--2005. Available at: https://www.cdc. gov/mmwr/preview/mmwrhtml/mm5508a3.htm. Accessed on 01 September 2019.

5. Lamas GA, Goertz C, Boineau R, Mark DB, Rozema T, Nahin RL, et al. Design of the trial to assess chelation therapy (TACT). Am Heart J. 2012;163:712.

6. Flora SJS, Shrivastava R, Mittal M. Chemistry and pharmacological properties of some natural and synthetic antioxidants for heavy metal toxicity, Curr Med Chem. 2013;20:4540-74.

7. Drisko JA. Chapter 107: Chelation therapy: in Integrative medicine. USA; Philadelphia: Elsevier; 2018.

8. American College of Cardiology - Chelation Therapy for CAD. Available at: https://www.acc.org/latest-incardiology/articles/2016/02/26/09/34/chelationtherapy-for-cad. Accessed on 01 September 2019.
9. Waters RS, Bryden NA, Patterson KY, Veillon C, Anderson RA. EDTA chelation effects on urinary losses of cadmium, calcium, chromium, cobalt, copper, lead, magnesium, and zinc. Biol Trace Elem Res. 2001;83:207-21.

10. Rekab K, Lepeytre C, Goettmann F, Dunand M, Guillard C, Hermann JM. Degradation of a cobalt(II)EDTA complex by photocatalysis and H2O2/UV-C. Application to nuclear wastes containing 60Co. J Radioanal Nucl Chem. 2014;303:131-7.

11. Peguero JG, Arenas I, Lamas GA. Chelation therapy and cardiovascular disease: Connecting scientific silos to benefit cardiac patients. Trends Cardiovasc Med. 2014; 24:232-40.

12. National Center for Complementary and Integrative Health - Questions and Answers: The NIH Trials of EDTA Chelation Therapy for Coronary Heart Disease. Available at: https://nccih.nih.gov/ health/chelation/TACT-questions. Accessed on 01 September 2019.

13. Wu SY, Tsai HC, Peng S Chang LT. Calciumactivated gene transfection from DNA/poly(Amic acid-co-imide) complexes. Int $\mathrm{J}$ Nanomedicine. 2015;10:1-11.

14. Seely DMR, Wu P, Mills EJ. EDTA chelation therapy for cardiovascular disease: a systematic review. BMC Cardio Dis. 2005;5:32.

15. Roussel AM, Favier IH, Waters R, Osman M, Fernholz K, Anderson RA. EDTA chelation therapy, without added vitamin $\mathrm{C}$, decreases oxidative DNA damage and lipid peroxidation. Altern Med Rev. 2009; 14:56-61.

16. The Role of Lipids and Lipoproteins in Atherosclerosis. Available at: https://www.ncbi.nlm. nih.gov/books/NBK343489/\#_ncbi_dlg_citbx_NBK3 43489. Accessed on 01 September 2019.

17. Escolar E, Lamas Gervasio A., Mark Daniel B., Boineau Robin, Goertz Christine, Rosenberg Yves, et al. The Effect of an EDTA-based Chelation Regimen on Patients With Diabetes Mellitus and Prior Myocardial Infarction in the Trial to Assess Chelation Therapy (TACT). Circ Cardiovasc Qual Outcomes. 2014;7:15-24.

18. Kraml P. The role of iron in pathogenesis of atherosclerosis. Physiol Res. 2017;66:S55-S67.

19. Synthesis of iron (III) EDTA complex Disease. Available at: https://www.stem.org.uk/resources/ elibrary/resource/35088/synthesis-ironiii-edtacomplex-nafeedta3h2o. Accessed on 01 September 2019.

20. Green DJ, O’Driscoll JG, Maiorana A, Serimgeour NB, Weerasooriya R, Taylor RR. Effects of chelation with EDTA and vitamin B therapy on nitric oxiderelated endothelial vasodilator function. Clin Exp Pharmacol P. 1999;26:853-856.

21. Flora SJS, Pachauri V. Chelation in metal intoxication, Int $\mathrm{J}$ Environ Res Public Health. 2010;7:2745-88. 
22. Chisolm J. The use of chelating agents in the treatment of acute and chronic lead intoxication in childhood. J Pediatr. 1968;73:1-38.

23. AHFS Drug information 86. Bethesda, MD: American Society of Hospital Pharmacists, 1986.

24. Gilman AG, Rall TW, Nies AS, Taylor P. Goodman and Gilman's the pharmacological basis of therapeutics. New York: Pergamon Press; 1990.

25. Anderson TJ, Hubacek J, Wyse Dg, Knudtson ML. Effect of chelation therapy on endothelial function in patients with coronary artery disease: PATCH substudy. J Am Coll Cardiol N Y. 2003;41:420-5.

26. Dans AL, Tan FN, Villarruz-Sulit EC. Chelation therapy for atherosclerotic cardiovascular disease (Review). Cochrane. 2002: 1-37.

27. Olszewer E, Sabbag FC, Carter JP. A pilot doubleblind study of sodium magnesium EDTA in peripheral vascular disease. J National Med Assoc. 1990;82:173-7.

28. Knudtson MI, Wyse DG, Galbraith PD, Brant R, Hildebrand K, Paterson D, et al. Chelation therapy for ischemic heart disease: A randomized controlled trial. JAMA. 2002;287:481-6.

29. Lamas GA, Goertz C, Boineau R, Mark DB, Rozema T, Nahin RL, et al. Effect of disodium EDTA chelation regimen on cardiovascular events in patients with previous myocardial infarction: the TACT randomized trial. JAMA. 2013;309:1241-50.

30. Spruance SL, Reid JE, Grace M, Samore M. Hazard ratio in clinical trials. Antimicrob Agents Chemother. 2004;48:2787-92.
31. Ujueta F, Arenas IA, Escolar E, Diaz D, Boineau R, Mark DB, et al. The effect of EDTA-based chelation on patients with diabetes and peripheral artery disease in the Trial to Assess Chelation Therapy (TACT). J Diabetes Complications. 2019;33:490-4.

32. Lamas GA, Boineau R, Goertz C, Mark DB, Rosenberg Y, Stylianou M, et al. EDTA chelation therapy alone and in combination with oral high-dose multivitamins and minerals for coronary disease: The factorial group results of the Trial to Assess Chelation Therapy. Am Heart J. 2014;168:37-44.e5.

33. Issa OM, Roberts R, Mark DB, Boineau R, Goertz C, Rosenberg $\mathrm{Y}$, et al. Effect of high-dose oral multivitamins and minerals in participants not treated with statins in the randomized Trial to Assess Chelation Therapy (TACT). Am Heart J. 2018;195:70-7.

34. Mathew RO, Schulman-Marcus J, Nichols EL, Newman JD, Bangalore S, Farkouh $M$, et al. Chelation therapy as a cardiovascular therapeutic strategy: The rationale and the data in review. Cardiovasc Drugs Ther. 2017;31:619-25.

35. Trial to Assess Chelation Therapy 2 (TACT2). Available at: https://clinicaltrials.gov/ct2/ show/NCT02733185. Accessed on 01 September 2019.

Cite this article as: Putra ICS, Kamarullah W. Diving deep into chelation therapy for coronary artery disease: a review. Int J Basic Clin Pharmacol 2019;8:2769-76. 